\title{
Le abitudini al tempo del Coronavirus
}

\author{
David Mariani ${ }^{1}$, Raffaele Picco ${ }^{2}$, Francesca Capitanini ${ }^{3}$, Alex Porciani ${ }^{4}$, Marco Lombardi ${ }^{5}$, Luigi Capotondo ${ }^{6}$, \\ Alessandro Capitanini ${ }^{7}$
}

${ }^{1}$ Healthy Habits Academy Montecatini Terme, Pistoia - Italia

${ }^{2}$ Nutrizionista e farmacista, Romentino, Novara - Italia

${ }^{3}$ Facoltà di Medicina Università degli Studi di Firenze, Firenze - Italia

${ }^{4}$ Ingegnere informatico, libero professionista, Zurigo - Svizzera

${ }^{5}$ SOS Nefrologia e Dialisi, Ospedale del Mugello, ASL Toscana Centro, Firenze - Italia

${ }^{6} \mathrm{Già} \mathrm{nefrologo} \mathrm{presso} \mathrm{l'Azienda} \mathrm{Ospedaliero-Universitaria} \mathrm{di} \mathrm{Siena,} \mathrm{Siena} \mathrm{-} \mathrm{Italia}$

${ }^{7} \mathrm{SOC}$ Nefrologia e Dialisi Ospedale San Jacopo, Pistoia, e ASL Toscana Centro, Firenze - Italia

\begin{abstract}
Habits in the time of Coronavirus
Introduction: The COVID-19 pandemic that hit the humankind in December 2019 is steering quick and drastic changes to our habits. The goal of our research is the analysis of the emotional, healthy and physiological effects of this radical routine disruption, in a sample of 3000 Italian people.

Methods: We made use of a 5-days flash survey in an anonymous way, available from April 5th until April 10th.

Results: As expected, the results show a decrease in health, after just one month of lockdown, at several stages: emotional, relational, nutritional and physical.

Conclusions: This quarantine period can be considered as an extreme example of immediate sedentary and isolation effects on people. Home habits such as basic physical activity, circadian rhythm routine, proper diet, and correct information consumption can be useful to increase our resilience in difficult times like the current one, but also in our next future.
\end{abstract}

Keywords: COVID-19, Exercise, Healthy habits, Nutrition, Psychology, Sedentary lifestyle, Social distancing

\section{Introduzione}

Dicembre 2019: in Cina, precisamente nella città di Wuhan, comincia a essere diagnosticato un alto numero di infezioni respiratorie acute. Si tratta di polmoniti con caratteristiche diverse da quelle abituali.

Ben presto, questo focolaio si espande e pian piano si diffonde anche in Europa, mettendo in ginocchio Spagna, Francia, Germania e molti altri paesi, in primis la nostra Italia.

È una situazione mai vissuta dai tempi della guerra. In poco più di 2 mesi si parla di "Pandemia" e diamo un nome al responsabile di queste strane polmoniti: Coronavirus 19, in gergo medico COVID-19.

Received: April 15, 2020

Accepted: April 16, 2020

Published online: May 6, 2020

Indirizzo per la corrispondenza:

David Mariani

Healthy Habits Academy

51016 Montecatini Terme, Pistoia - Italia

d.mariani@healthyhabits.it
Non appena comprese le caratteristiche di contagiosità e pericolosità per la salute umana di questa nuova infezione, le istituzioni nazionali si sono viste costrette, nel tentativo di contenere la diffusione della patologia, a porre in atto fortissime limitazioni su spostamenti, contatti e libertà personale. Questo, chiaramente, ha provocato un cambiamento repentino e di grande impatto sulle abitudini di vita di milioni di persone.

L'indagine alla base di questo lavoro si propone di analizzare gli effetti che questo vero e proprio stravolgimento della nostra routine ha prodotto a livello emotivo, fisico e fisiologico e ci lascia intravedere, come fosse un trailer, le conseguenze di una vita fortemente sedentaria.

In Italia, infatti, i DPCM (Decreti del Presidente del Consiglio dei Ministri) hanno, via via, aumentato i divieti atti a regolamentare le uscite e gli spostamenti, anche nell'ambito della propria provincia, arrivando a sconsigliare, per non dire a vietare, la pratica di qualunque attività fisica all'aperto e autorizzando soltanto gli spostamenti per motivi di salute e cause di forza maggiore: la situazione in cui la maggior parte della popolazione si è venuta a trovare è stata quella di dover trascorrere settimane barricata nella propria abitazione.

Prendiamo, dunque, in esame l'impatto che questa situazione può determinare sulla salute umana. 
In primis, il risvolto concreto di questa situazione è stato il passaggio (o il proseguimento) a una vita più sedentaria. Dal punto di vista fisiopatologico, la sedentarietà risulta essere uno dei più grandi mali dell'umanità, tanto da essere indicata come il quarto fattore di mortalità precoce dall'OMS (1).

Lo stare fermi, seduti per ore, produce effetti veramente pericolosi per l'organismo umano: basti pensare che 8 ore di immobilità quotidiane, su una sedia o su un divano, sono in grado di ridurre la durata media della vita quotidiana di quattro anni (2).

Se è vero, infatti, che l'esercizio fisico è il farmaco più potente (ed economico) mai inventato, in grado di intervenire in via preventiva su moltissime patologie, è altrettanto vero che privarsene vuol dire, per contro, dare il via libera a una serie di disfunzioni che riguardano l'organismo in tutti i suoi apparati.

La sedentarietà non è solo un fattore predittivo di mortalità, ma anche un potente acceleratore di tutta una serie di cattivi comportamenti, ben identificati sia dal punto di vista sociale che fisiologico (3). La ricerca scientifica ha, infatti, evidenziato che le persone sedentarie sono più esposte al rischio di avere il vizio del fumo, consumare alte dosi di alcolici, abusare del cibo e avere peggiori relazioni sociali.

La permanenza nella propria abitazione, poi, oltre a renderci più sedentari, ci spinge ad abusare della tecnologia, con il conseguente uso eccessivo di dispositivi come tv, computer e smartphone che, come sappiamo, emettono toni di luce molto diversi da quelli a cui l'essere umano è abituato da millenni.

Inoltre, sono ormai quasi sempre questi dispositivi a tenerci informati su ciò che succede nel mondo: la conseguenza di questa continua informazione, o dis-informazione a seconda dei casi, è anche quella di esporci a un continuo bombardamento di pessimismo, cronaca nera e paura.

Oltre ai mancati benefici dell'attività fisica, dunque, lo stress e la paura derivanti da questa situazione e dalla comunicazione mediatica, che tende, per motivi di audience, a drammatizzare al massimo la situazione, provocano come reazione organica l'immissione in circolo di ormoni stressanti nell'organismo, soprattutto cortisolo e adrenalina. L'aumento di questi ormoni porta a un incremento della frequenza cardiaca e della pressione arteriosa e anche a un aumentato bisogno di zuccheri. Come se non bastasse, a ciò si aggiunge la tendenza ad avere un sonno più leggero e meno riposante (4), utile nelle situazioni in cui siamo sotto attacco, per poter essere più vigili e reattivi, sì, ma molto meno se questa situazione si verifica in tempi di pace o, comunque, in situazioni come questa.

Questi sono gli spunti di riflessione che ci hanno spinto a indagare gli effetti della pandemia sui comportamenti della popolazione e lo abbiamo fatto attraverso un'indagine digitale, facendo compilare anonimamente un questionario composto da domande in grado di esplorare ognuno degli aspetti che abbiamo ritenuto più importanti: emotività, qualità del sonno, relazioni sociali, dieta e attività fisica.

\section{Materiali e metodi}

Abbiamo utilizzato il survey provider di Google (gratuito), "Google Forms", per realizzare una flash survey della durata di 5 giorni tramite un questionario anonimo inviato attraverso gruppi di WhatsApp e di Facebook e aperto dal 5 al 10 aprile 2020.

Nel questionario, oltre alle generalità (sesso, fascia d'età, attività professionale in atto o interrotta), abbiamo analizzato alcuni ambiti della vita delle persone: sfera emotiva, sfera relazionale, sfera fisiologica, sfera nutrizionale attività fisica (Allegato 1).

\section{Risultati}

Complessivamente, sono stati completati 3.021 questionari. II 76\% dei questionari è stato compilato da persone di sesso femminile e il 59,6\% dei soggetti ha dichiarato di avere un'età compresa tra i 30 e i 60 anni. II 34,9\% dei soggetti afferma di aver dovuto interrompere il proprio lavoro e il 18,1\% sostiene di averlo convertito in smart working da remoto.

II 42\% degli individui analizzati risulta aver sperimentato un peggioramento della qualità del proprio sonno, con un peggioramento del proprio stato emotivo nel $52,4 \%$ dei casi.

Le relazioni sociali sono peggiorate per il 56,8\% degli intervistati e circa l' $80 \%$ di loro ha aumentato il tempo trascorso davanti a un monitor.

Oltre la metà dei soggetti ha, poi, aumentato la quantità di cibo, con il 39\% di aumento alla voce zuccheri (dolci, zucchero, pane e pasta); infine, oltre la metà dei soggetti ha ridotto la quantità della propria attività fisica (Allegato 2 ).

\section{Discussione}

Complessivamente, la spinta all'isolamento e alla sedentarietà rappresentano condizioni che vanno contro la nostra storia evolutiva, durata milioni di anni, caratterizzata, piuttosto, dalla tendenza all'unione e alla cooperazione. Come sappiamo, l'andare contro la propria natura ha profonde ripercussioni negative sulla fisiologia umana e, probabilmente, risentiremo di questo brusco e profondo mutamento per un lungo periodo.

Ma quali spunti possiamo trarre dai dati raccolti con il questionario?

Analizziamo alcune sfere fondamentali della vita dell'uomo: attività fisica, nutrizione, sfera psichica e relazionale e condizioni ambientali.

L'imposizione di restare a casa ha favorito la sedentarietà, come testimoniano le risposte dei nostri partecipanti. Uno dei modi per mitigare i danni della sedentarietà sarebbe fare ginnastica in casa. La cosa non è facile, soprattutto per chi vive in spazi molto limitati. In questo caso, però, proprio i social e la tecnologia, con cui vige un rapporto di amoreodio, ci sono giunti in soccorso con la rapida moltiplicazione e 
diffusione di tutorial (danza, ginnastica, yoga...) prodotti dalle varie associazioni sportive e non (palestre, piattaforme televisive, istituzioni).

Comunque, anche per chi non abbia possibilità o voglia di seguire tutorial, consigliamo di praticare quotidianamente almeno 20 minuti (meglio 30-40) di esercizio fisico, anche diviso in due sessioni. Come? Possiamo fare esercizio aerobico, per esempio la marcia sul posto sollevando bene le ginocchia, possibilmente in giardino o in terrazza per aumentarne i benefici grazie alla luce solare, oppure rimanere dentro casa facendo semplici esercizi come squat o accosciata, mettendo una sedia dietro di noi o stando in piedi davanti al letto per evitare cadute, soprattutto se siamo un po' avanti con gli anni (e, per questo, anche più bisognosi di esercizio) (5).

Anche le abitudini alimentari hanno risentito negativamente di questa situazione di isolamento: le preoccupazioni, ma anche la "noia", hanno portato alla ricerca di appagamento nel cibo per una sorta di ricompensa psicologica in grado di fungere da ansiolitico. In particolare, i carboidrati hanno giocato la parte del leone. Chiaramente mangiare di più in un momento di consumo minore non rappresenta la scelta migliore da fare.

A livello nutrizionale, anche per potenziare le difese immunitarie, potremmo: sostituire gli zuccheri semplici, gli alimenti trasformati, i cibi industriali, il cibo spazzatura, i fast food e l'eccesso di cibo animale con cibi di origine vegetale, non raffinati e ricchi di fibra e di nutrienti "veri". È sufficiente, quindi, mangiare pane integrale al posto di pane bianco e riso integrale al posto di riso bianco e riscoprire i cosiddetti "cereali antichi", quelli che, per secoli, hanno dato forza ed energia ai nostri predecessori, come farro, avena, orzo e grano saraceno (6).

Un ruolo fondamentale è quello delle fibre, che, nutrendo la nostra flora batterica, mantengono in condizioni di efficienza il nostro intestino e mantengono, di conseguenza, il nostro sistema immunitario vigile e attento (7-9).

Ottimale sarebbe anche ridurre i livelli di zucchero che introduciamo quotidianamente e, da qui, l'invito a diminuire più che possiamo farina 00 , dolciumi commerciali e cereali raffinati (che hanno un alto indice glicemico) (10).

Grandi studi scientifici (11-13) hanno anche dimostrato che il consumo abbondante di carne incrementa molto il rischio di contrarre malattie infettive e che un eccesso proteico di derivazione animale stressa eccessivamente il nostro organismo.

Cerchiamo, dunque, di iniziare ad apprezzare le fonti proteiche vegetali, per esempio legumi e frutta secca $(14,15)$.

Ultimo trucco per capire se il nostro pranzo e la nostra cena sono sani e positivi per noi è la semplice osservazione: se, a occhio nudo, la verdura supera per quantità tutte le altre portate del pasto, siamo sulla strada giusta e il nostro corpo ne trarrà grande giovamento.

Mantenere e migliorare le nostre abitudini, organizzando le nostre giornate, è, poi, fondamentale al fine di evitare che noia e scoraggiamento prendano il sopravvento su di noi, creando un circolo vizioso che ci porterà ad aumentare, giorno dopo giorno, negatività, stress e malessere.

Ecco una possibilità di strutturare in modo diverso le vostre giornate:

- Evitate di stare in pigiama.

- Alzatevi come al solito o, comunque, non troppo tardi.

- Rispettate gli orari corretti dei pasti e chi, a causa del lavoro, saltava spesso il pranzo o lo preparava velocemente con cibo di scarsa qualità approfitti per migliorare la qualità dei propri pasti (16).

- Rispettate i giusti ritmi di sonno e veglia, evitando di andare a dormire troppo tardi la sera, per non incappare in un'eccessiva sregolatezza che si ripercuoterà sulle vostre abitudini quotidiane (17).

- Pianificate il lavoro da casa e altre attività, in modo tale da mantenere sempre il controllo del vostro tempo.

- Utilizzate i media in modo consapevole, ascoltando fonti affidabili, senza, però, andare alla ricerca compulsiva di informazioni: un telegiornale al giorno può bastare. Non nutriamoci di negatività (18).

- Riscoprite i vostri punti di forza, perché prendere coscienza delle vostre risorse interiori vi aiuterà a superare i momenti di crisi. Chiudete la porta alla paura (che paralizza) e spalancatela alla speranza (che non è illusione e rivitalizza).

- Leggete libri messi da parte per mancanza di tempo, approfittate per curiosare online nelle numerose biblioteche che hanno messo in rete il proprio catalogo di titoli, trasformate la lettura in un passatempo per tutta la famiglia. Dipingete, cantate e ascoltate buona musica senza disturbare i vicini.

Insomma fate ciò che più vi piace e stimola, dedicandovi alle cose a cui, prima, troppo spesso avete rinunciato proprio per la solita "mancanza di tempo".

Il distanziamento sociale ha evidenziato la necessità umana della vicinanza: i canti fuori dalla finestra, le video chat e la fratellanza all'interno dei condomini probabilmente ci hanno avvicinato, seppur a distanza di sicurezza, gli uni agli altri.

Da qui, la consapevolezza che "non tutto il male viene per nuocere". La brutta esperienza di questa pandemia può essere utilizzata per farci aprire gli occhi su ciò che conta davvero e per migliorare le nostre abitudini.

Insieme possiamo farcela... anche stando a casa come ci viene ora richiesto.

\section{Disclosures}

Conflict of interest: The authors have no conflict of interest. Financial support: This research received no specific grant from any funding agency in the public, commercial, or not-for-profit sectors. 


\section{Bibliografia}

1. WHO. Global health risks. Mortality and burden of disease attributable to selected major risks. 2009. http://www.who.int/ healthinfo/global_burden_disease/GlobalHealthRisks_report_ full.pdf (accessed Jan 15, 2012).

2. Ekelund U, Steene-Johannessen J, Brown WJ, et al. Does physical activity attenuate, or even eliminate, the detrimental association of sitting time with mortality? A harmonised metaanalysis of data from more than 1 million men and women. Lancet. 2016;388(10051):1302-10.

3. Scarborough P, Bhatnagar P, Wickramasinghe KK, Allender S, Foster C, Rayner M. The economic burden of ill health due to diet, physical inactivity, smoking, alcohol and obesity in the UK: an update to 2006-07 NHS costs. J Public Health (Oxf). 2011;33:527-35.

4. Russell G, Lightman S. The human stress response. Nat Rev Endocrinol. 2019;15:525-34.

5. Capitanini A, Mariani D. Born to run... ma anche percamminare. Attività motoria, il primo pilastro dell'evoluzione umana. Giornale di Tecniche Nefrologiche e Dialitiche. 2019;31(2): 142-5.

6. Mozaffarian RS, Lee RM, Kennedy MA, Ludwig DS, Mozaffarian D, Gortmaker SL. Identifying whole grain foods: a comparison of different approaches for selecting more healthful whole grain products. Public Health Nutr. 2013;16:2255-64.

7. Cario E. Bacterial interactions with cells of the intestinal mucosa: Toll-like receptors and NOD2. Gut. 2005;54:1182-93. doi: 10.1136/gut.2004.062794.

8. Ramberg JE, Nelson ED, Sinnott RA. Immunomodulatory dietary polysaccharides: A systematic review of the literature. Nutr J. 2010;9:54.
9. Hosseinpour-Niazi S, Mirmiran P, Sohrab G, et al. Inverse association between fruit, legume, and cereal fiber and the risk of metabolic syndrome: Tehran Lipid and Glucose Study. Diabetes Res Clin Pract. 2011;94:276-83.

10. Ludwig DS, Majzoub JA, Al-Zahrani A, Dallal GE, Blanco I, Roberts SB. High glicemic index foods, overeating, and obesity. Pediatrics. 1999;103:E26.

11. López-Gómez JJ, Izaola-Jauregui O, Primo-Martín D, et al. Effect of Two Meal Replacement strategies on Cardiovascular Risk Parameters in Advanced Age Patients with Obesity and Osteoarthritis. Nutrients. 2020;12:976.

12. Sinha $R$, Cross AJ, Graubard BI, Leitzmann MF, Schatzkin A. Meat intake and mortality: A prospective study of over half a million people. Arch Intern Med. 2009;169(6):562-71.

13. Larsson SC, Orsini N. Red meat and processed meat consumption and all-cause mortality: a meta-analysis. Am J Epidemiol. 2014;179(3):282-9.

14. Rui-Hai L. Health-promoting components of fruits and vegetables in the diet. Adv Nutr. 2013;4(3):384S-392S.

15. Martinez-Gonzalez MA, Salas-Salvado J, Estruch R, et al. Benefits of the Mediterranean diet: insights from the PREDIMED Study. Prog Cardiovasc Dis. 2015;58:50-60.

16. Jayalath VH, de Souza RJ, Sievenpiper JL, et al. Effect of dietary pulses on blood pressure: a systematic review and meta-analysis of controlled feeding trials. Am J Hypertens. 2014;27:56-64.

17. von Ruesten A, Weikert C, Fietze I, Boeing H. Association of sleep duration with chronic diseases in the European Prospective Investigation into Cancer and Nutrition (EPIC)-Potsdam study. PLoS One. 2012;7:e30972.

18. Ross JG, Myers SM. The Current Use of Social Media in Undergraduate Nursing Education: A Review of the Literature. Comput Inform Nurs. 2017;35(7):338-44. 